\title{
A New Approach to the Solution of the Fredholm Integral Equation via a Fixed Point on Extended $b$-Metric Spaces
}

\author{
Erdal Karapınar ${ }^{1,2}$, Panda Sumati Kumari ${ }^{3}$ (i) and Durdana Lateef ${ }^{4, *}$ \\ 1 Department of Mathematics, Atilim University, İncek, 06836 Ankara, Turkey; erdalkarapinar@yahoo.com \\ 2 Department of Medical Research, China Medical University, Taichung 40402, Taiwan \\ 3 Department of Mathematics, Basic Sciences and Humanities, GMR Institute of Technology, Andhra Pradesh, \\ Rajam 532127, India; mumy143143143@gmail.com \\ 4 Department of Mathematics, College of Science, Taibah University, Al Madina Al Munawwara, \\ Madina 41411, Saudi Arabia \\ * Correspondence: drdurdanamaths@gmail.com
}

Received: 7 September 2018; Accepted: 13 October 2018; Published: 16 October 2018

\begin{abstract}
It is very well known that real-life applications of fixed point theory are restricted with the transformation of the problem in the form of $f(x)=x$. (1) The Knaster-Tarski fixed point theorem underlies various approaches of checking the correctness of programs. (2) The Brouwer fixed point theorem is used to prove the existence of Nash equilibria in games. (3) Dlala et al. proposed a solution for magnetic field problems via the fixed point approach.

In this paper, by obtaining the fixed point results in an extended $b$-metric space, we are able to consider real-life applications in a very general frame such as a simple and efficient solution for a Fredholm integral equation by using the technique of a fixed point in the consideration of a new abstract space: the extended $b$-metric space. Moreover, to address conceptual depth within this approach, we supply illustrative examples of usage where necessary.
\end{abstract}

Keywords: extended $b$-metric space; extended cyclic orbital contraction; extended cyclic orbital- $\mathcal{F}$-contraction and Fredholm integral equation

\section{Introduction}

In 1903, Erik Ivar Fredholm [1] introduced a revolutionary result in the theory of nonlinear integral equations, and it was defined by the fixed limits of integration of the expression:

$$
\kappa(x)=\Lambda(x)+\lambda \int_{p}^{q} \Xi(x, t) u(t) d t
$$

where the terms $p$ and $q$ are denoted as constants, $\lambda$ is a parameter, $\Lambda(x)$ is the data function, $\Xi(x, t)$ is called the kernel function and $\kappa(x)$ is the unknown function.

Fredholm integral equations appear widely in many scientific areas like physical mathematics, computational mathematics and approximation theory.

Recently, several authors have extensively studied the solution of the Fredholm integral equation via the fixed point approach (see, e.g., [2-5]).

The Banach contraction principle is a one of the superior results in non-linear analysis and has always been at the forefront of creating and supplying outstanding generalizations for its researchers. Many authors have generalized and utilized the Banach contraction principle in their pertinent research. Thus, we can easily conclude that the largest part of the fixed point theory has been occupied by various generalizations of the Banach contraction principle. 
Throughout the paper, $\mathbb{R}, \mathbb{R}^{+}, \mathbb{N}$ stand for the reals, positive reals and natural numbers, accordingly. Moreover, we employ the symbols $\mathbb{R}_{0}^{+}=\mathbb{R}^{+} \cup\{0\}=[0, \infty)$ and $\mathbb{N}_{0}=\mathbb{N} \cup\{0\}$. Below are some of the standard extensions of the Banach contraction principle.

Cyclic contraction by Kirk et al. [6] $\Leftrightarrow$ There exists $k \in(0,1)$ such that:

$$
d(\mathcal{Z} x, \mathcal{Z} y) \leq k d(x, y) \text { for all } x \in \mathbb{S}_{1} \text { and } y \in \mathbb{S}_{2}
$$

(Note that, a mapping $\mathcal{Z}: \mathbb{S}_{1} \cup \mathbb{S}_{2} \rightarrow \mathbb{S}_{1} \cup \mathbb{S}_{2}$ is called cyclic if $\mathcal{Z}\left(\mathbb{S}_{1}\right) \subseteq \mathbb{S}_{2}$ and $\mathcal{Z}\left(\mathbb{S}_{2}\right) \subseteq \mathbb{S}_{1}$, where $\mathbb{S}_{1}, \mathbb{S}_{2}$ are nonempty subsets of a metric space $(M, d)$.)

Cyclic orbital contraction by Karpagam et al. [7] $\Leftrightarrow d\left(\mathcal{Z}^{2 n} x, \mathcal{Z} y\right) \leq \gamma d\left(\mathcal{Z}^{2 n-1} x, y\right)$;

for all $x \in \mathbb{S}_{1}, \gamma \in(0,1)$; where $\mathbb{S}_{1}$ and $\mathbb{S}_{2}$ are non-empty closed subsets of $M$ and $\mathcal{Z}: \mathbb{S}_{1} \cup \mathbb{S}_{2} \rightarrow$ $\mathbb{S}_{1} \cup \mathbb{S}_{2}$ is a cyclic map.

$\mathcal{F}$-contraction by Wardowski [8] $\Leftrightarrow$ There is a $\tau>0$ such that for all $x, y \in M$,

$$
d(\mathcal{Z} x, \mathcal{Z} y)>0 \Rightarrow \tau+F(d(\mathcal{Z} x, \mathcal{Z} y)) \leq F(d(x, y))
$$

where $F: \mathbb{R}_{0}^{+} \rightarrow \mathbb{R}$ is a mapping satisfying

$(F 1) . F$ is strictly increasing, which claims, for all $t_{1}, t_{2} \in \mathbb{R}_{0}^{+}$such that if $t_{1}<t_{2}$, then $F\left(t_{1}\right)<F\left(t_{2}\right)$;

$(F 2)$. For each sequence $\left\{t_{1_{n}}\right\}_{n \in \mathbb{N}}$ of positive numbers:

$$
\lim _{n \rightarrow \infty} t_{1_{n}}=0 \text { iff } \lim _{n \rightarrow \infty} F\left(t_{1_{n}}\right)=-\infty,
$$

(F3), there exists $k \in(0,1)$ such that $\lim _{t_{1} \rightarrow 0^{+}} t_{1}^{k} F\left(t_{1}\right)=0$.

We represent by $\mathcal{F}$ the set of all functions satisfying the above mentioned conditions.

For more literature pertinent to above, the reader can refer to [6-19].

Recently, a new kind of generalized metric space was introduced by T.Kamran et al. [14], announced as the $E_{b}$-metric space.

Definition 1. Let $M$ be a non-empty set and $s: M \times M \rightarrow[1, \infty)$. A function $E_{b}: M \times M \rightarrow[0, \infty)$ is called an $E_{b}$-metric if, for all $x, y, z \in M$, it satisfies:

(i) $E_{b}(x, y)=0$ if and only if $x=y$;

(ii) $E_{b}(x, y)=E_{b}(y, x)$;

(iii) $E_{b}(x, y) \leq s(x, y)\left[E_{b}(x, z)+E_{b}(z, y)\right]$.

The pair $\left(M, E_{b}\right)$ is called an $E_{b}$-metric space.

It is clear that if $s(x, y)$, in Definition 1 , is constant in $[1, \infty)$, the pair $\left(M, E_{b}\right)$ coincides with the $b$-metric space.

Example 1. Let $M=\{1,2,3\}$. Define $s: M \times M \rightarrow[1, \infty)$ and $E_{b}: M \times M \rightarrow[0, \infty)$ as $s(x, y)=1+x y$ and:

$$
\begin{gathered}
E_{b}(1,1)=E_{b}(2,2)=E_{b}(3,3)=0 ; \\
E_{b}(1,2)=E_{b}(2,1)=2 ; \\
E_{b}(1,3)=E_{b}(3,1)=100 ; \\
E_{b}(2,3)=E_{b}(3,2)=50 .
\end{gathered}
$$

Clearly, (i) and (ii) hold. For (iii), we have:

$$
E_{b}(1,2)=2 ; s(1,2)\left[E_{b}(1,3)+E_{b}(3,2)\right]=450 .
$$


Thus,

$$
\begin{gathered}
E_{b}(1,2) \leq s(1,2)\left[E_{b}(1,3)+E_{b}(3,2)\right] . \\
E_{b}(1,3)=100 ; s(1,3)\left[E_{b}(1,2)+E_{b}(2,3)\right]=208 .
\end{gathered}
$$

Then,

$$
\begin{gathered}
E_{b}(1,3) \leq s(1,3)\left[E_{b}(1,2)+E_{b}(2,3)\right] . \\
E_{b}(2,3)=50 ; s(2,3)\left[E_{b}(2,1)+E_{b}(1,3)\right]=714 .
\end{gathered}
$$

Therefore,

$$
E_{b}(2,3) \leq s(2,3)\left[E_{b}(2,1)+E_{b}(1,3)\right] .
$$

Hence, for all $x, y, z \in M$, we have $E_{b}(x, z) \leq s(x, z)\left[E_{b}(x, y)+E_{b}(y, z)\right]$. Consequently, the pair $\left(M, E_{b}\right)$ forms an $E_{b}$-metric space.

Definition 2. (See, e.g., [14]) Let $\left(M, E_{b}\right)$ be an $E_{b}$-metric space, and a sequence $\left\{x_{n}\right\}$ in $M$ is said:

(a) to converge to $x$ if and only if for every $\epsilon>0$, there exists $N=N(\epsilon) \in \mathbb{N}$ such that $E_{b}\left(x_{n}, x\right)<\epsilon$, for all $n \geq N$. For this particular case, we write $\lim _{n \rightarrow \infty} x_{n}=x$.

(b) to be Cauchy if and only if for every $\epsilon>0$, there exists $N=N(\epsilon) \in \mathbb{N}$ such that $E_{b}\left(x_{m}, x_{n}\right)<\epsilon$, for all $m, n \geq N$.

Definition 3. (See [14]) An $E_{b}$-metric space $\left(M, E_{b}\right)$ is complete if and only if every Cauchy sequence in $M$ is convergent.

Observe that usually, a $b$-metric is not a continuous functional. Analogously, the functional, $E_{b}$-metric, is also not necessarily a continuous functional (see, e.g., [15-17]).

Motivated by the above facts, we introduce and establish various approaches to cyclic orbital contraction using new sorts of contractions, named as extended cyclic orbital contraction and extended cyclic orbital- $\mathcal{F}$-contraction in the setting of an $E_{b}$-metric space. Thereafter, we propose a simple and efficient solution for a Fredholm integral equation by using the technique of the fixed point in the setting of the $E_{b}$-metric space. Moreover, to address conceptual depth within this approach, we supply illustrative examples of usage where necessary.

\section{An Extended Cyclic Orbital Contraction}

We start this section by introducing the notion of an extended cyclic orbital contraction.

Definition 4. Let us take two non-empty subsets $\mathbb{S}_{1}$ and $\mathbb{S}_{2}$ of a $E_{b}$-metric space $\left(M, E_{b}\right)$, and let $\mathcal{Z}: \mathbb{S}_{1} \cup \mathbb{S}_{2} \rightarrow$ $\mathbb{S}_{1} \cup \mathbb{S}_{2}$ be a cyclic map such that for some $x \in \mathbb{S}_{1}$, there exists a $k_{x} \in(0,1)$ such that:

$$
E_{b}\left(\mathcal{Z}^{2 n} x, \mathcal{Z} y\right) \leq k_{x} E_{b}\left(\mathcal{Z}^{2 n-1} x, y\right)
$$

where $n \in \mathbb{N}, y \in \mathbb{S}_{1}$. Then, $\mathcal{Z}$ is called an extended cyclic orbital contraction.

Theorem 1. Let $E_{b}$ be a continuous functional in complete $E_{b}$-metric space $\left(M, E_{b}\right)$. Let $\mathbb{S}_{1}$ and $\mathbb{S}_{2}$ be non-empty subsets of an $E_{b}$-metric space $\left(M, E_{b}\right)$ and $\mathcal{Z}: \mathbb{S}_{1} \cup \mathbb{S}_{2} \rightarrow \mathbb{S}_{1} \cup \mathbb{S}_{2}$ be an extended cyclic orbital contraction. Suppose that for each $x_{0} \in \mathbb{S}_{1}, \lim _{n, m \rightarrow \infty} s\left(x_{n}, x_{m}\right)<\frac{1}{k_{x_{0}}}$; here, $x_{n}=\mathcal{Z}^{n} x_{0} ; n=1,2,3 \ldots$ Then, $\mathbb{S}_{1} \cap \mathbb{S}_{2}$ is non-empty, and $\mathcal{Z}$ has a unique fixed point.

Proof. Suppose there exists an $x$ (say $\left.x_{0}\right), \in \mathbb{S}_{1}$ satisfying (2). Define an iterative sequence $\left\{x_{n}\right\}$ starting by $x_{0}$, as follows:

$$
\mathcal{Z} x_{0}=x_{1}, x_{2}=\mathcal{Z} x_{1}=\mathcal{Z}\left(\mathcal{Z} x_{0}\right)=\mathcal{Z}^{2}\left(x_{0}\right) \ldots \ldots x_{n}=\mathcal{Z}^{n}\left(x_{0}\right) \ldots
$$


On account of (2), we find that $E_{b}\left(\mathcal{Z}^{2} x, \mathcal{Z} x\right) \leq k_{x} E_{b}(\mathcal{Z} x, x)$.

Recursively, we derive that:

$$
\begin{aligned}
E_{b}\left(\mathcal{Z}^{3} x, \mathcal{Z}^{2} x\right) & \leq k_{x} E_{b}\left(\mathcal{Z}^{2} x, \mathcal{Z} x\right) \\
& \leq k_{x}^{2} E_{b}(\mathcal{Z} x, x)
\end{aligned}
$$

Since for any $n \in \mathbb{N}$, there is a possibility that either $n$ or $n+1$ is even, we have:

$$
E_{b}\left(\mathcal{Z}^{n+1} x, \mathcal{Z}^{n} x\right) \leq k_{x}^{n} E_{b}(\mathcal{Z} x, x),
$$

that is,

$$
E_{b}\left(x_{n+1}, x_{n}\right) \leq k_{x}^{n} E_{b}\left(x_{1}, x_{0}\right) .
$$

By the triangle inequality and (2), for $m>n$, we have:

$$
\begin{aligned}
E_{b}\left(\mathcal{Z}^{n} x, \mathcal{Z}^{m} x\right)= & E_{b}\left(x_{n}, x_{m}\right) \\
\leq & s\left(x_{n}, x_{m}\right) k_{x}^{n} E_{b}\left(x_{0}, x_{1}\right)+s\left(x_{n}, x_{m}\right) s\left(x_{n+1}, x_{m}\right) k_{x}^{n+1} E_{b}\left(x_{0}, x_{1}\right) \\
& +s\left(x_{n}, x_{m}\right) s\left(x_{n+1}, x_{m}\right) s\left(x_{n+2}, x_{m}\right) \ldots . . s\left(x_{m-2}, x_{m}\right) s\left(x_{m-1}, x_{m}\right) k_{x}^{m-1} E_{b}\left(x_{0}, x_{1}\right) \\
\leq & E_{b}\left(x_{0}, x_{1}\right)\left[s\left(x_{1}, x_{m}\right) s\left(x_{2}, x_{m}\right) \ldots s\left(x_{n-1}, x_{m}\right) s\left(x_{n}, x_{m}\right) k_{x}^{n}\right. \\
& +s\left(x_{1}, x_{m}\right) s\left(x_{2}, x_{m}\right) \ldots s\left(x_{n}, x_{m}\right) s\left(x_{n+1}, x_{m}\right) k_{x}^{n+1} \\
& \vdots \\
& +s\left(x_{1}, x_{m}\right) s\left(x_{2}, x_{m}\right) \ldots s\left(x_{m-2}, x_{m}\right) s\left(x_{m-1}, x_{m}\right) k_{x}^{m-1} .
\end{aligned}
$$

Since $\lim _{n, m \rightarrow \infty} s\left(x_{n+1}, x_{m}\right) k_{x}<1$, the series:

$$
\sum_{n=1}^{\infty} k_{x}^{n} \prod_{i=1}^{n} s\left(x_{i}, x_{m}\right)
$$

converges as per the ratio test for each $m \in \mathbb{N}$.

Let:

$$
S=\sum_{n=1}^{\infty} k_{x}^{n} \prod_{i=1}^{n} s\left(x_{i}, x_{m}\right) \text { and } S_{n}=\sum_{j=1}^{n} k_{x}^{j} \prod_{i=1}^{j} s\left(x_{i}, x_{m}\right) .
$$

Thus, for $m>n$, the above inequality implies:

$$
\begin{aligned}
E_{b}\left(\mathcal{Z}^{n} x, \mathcal{Z}^{m} x\right) & =E_{b}\left(x_{n}, x_{m}\right) \\
& \leq E_{b}\left(x_{0}, x_{1}\right)\left[S_{m-1}-S_{n-1}\right]
\end{aligned}
$$

Letting $n \rightarrow \infty$, we conclude that $\left\{\mathcal{Z}^{n} x\right\}$ is a Cauchy sequence, and as a result, there exists a $\rho \in \mathbb{S}_{1} \cup \mathbb{S}_{2}$ such that $\mathcal{Z}^{n} x \rightarrow \rho$. Now, note that $\left\{\mathcal{Z}^{2 n} x\right\}$ is a sequence in $\mathbb{S}_{1},\left\{\mathcal{Z}^{2 n-1} x\right\}$ is a sequence in $\mathbb{S}_{2}$ and both converge to $\rho$, since the sets $\mathbb{S}_{1}$ and $\mathbb{S}_{2}$ are closed in $M$ and $\rho \in \mathbb{S}_{1} \cap \mathbb{S}_{2}$. Hence, $\mathbb{S}_{1} \cap \mathbb{S}_{2}$ is non-empty.

To prove $\rho=\mathcal{Z} \rho$, consider:

$$
\begin{aligned}
E_{b}(\rho, \mathcal{Z} \rho) & =\lim _{n \rightarrow \infty} E_{b}\left(\mathcal{Z}^{2 n} x, \mathcal{Z} \rho\right) \\
& \leq k_{x} \lim _{n \rightarrow \infty} E_{b}\left(\mathcal{Z}^{2 n-1} x, \rho\right) \\
& =0
\end{aligned}
$$


Thus, $\rho$ is a fixed point of $\mathcal{Z}$. In order to get the uniqueness of $\rho$, assume there exists an $\varrho \in$ $\mathbb{S}_{1} \cup \mathbb{S}_{2}, \rho \neq \varrho$ such that $\mathcal{Z} \varrho=\varrho$.

Now,

$$
\begin{aligned}
E_{b}(\rho, \varrho) & =E_{b}(\rho, \mathcal{Z} \varrho) \\
& =\lim _{n \rightarrow \infty} E_{b}\left(\mathcal{Z}^{2 n} x, \mathcal{Z} \varrho\right) \\
& \leq k_{x} \lim _{n \rightarrow \infty} E_{b}\left(\mathcal{Z}^{2 n-1} x, \varrho\right) \\
& =k_{x} E_{b}(\rho, \varrho) \\
& <E_{b}(\rho, \varrho)
\end{aligned}
$$

Therefore, $\rho=\varrho$. Thus, $\rho$ is the unique fixed point of $\mathcal{Z}$. This completes the proof.

Example 2. Let $M=\mathbb{R}$. Define $E_{b}(x, y): M \times M \rightarrow \mathbb{R}_{0}^{+}$and $s: M \times M \rightarrow[1, \infty)$ as $E_{b}(x, y)=(x-y)^{2}$ and $s(x, y)=x+y+1$. Then, $E_{b}$ is a complete $E_{b}$-metric on $M$.

Let $\mathbb{S}_{1}=\{0,1\}, \mathbb{S}_{2}=\left\{0, \frac{13}{3}\right\}$. Define $\mathcal{Z}: \mathbb{S}_{1} \cup \mathbb{S}_{2} \rightarrow \mathbb{S}_{1} \cup \mathbb{S}_{2}$ by $\mathcal{Z} 0=\mathcal{Z} 1=0 ; \mathcal{Z} \frac{13}{3}=1$.

We have $\mathcal{Z} \mathbb{S}_{1} \subseteq \mathbb{S}_{2}$ and $\mathcal{Z} \mathbb{S}_{2} \subseteq \mathbb{S}_{1}$. Thus, $\mathcal{Z}$ is a cyclic map.

Fix $x=1$.

$\mathcal{Z} x=0, \mathcal{Z}^{2} x=\mathcal{Z}(0)=0, \mathcal{Z}^{3}=\mathcal{Z}(0)=0, \ldots . . \mathcal{Z}^{n}(x)=0$. Therefore, $\mathcal{Z}^{2 n}(x)=0=\mathcal{Z}^{2 n-1}(x)$.

Case I. If $y=0$, then $\mathcal{Z} y=0$.

Thus,

$$
\begin{aligned}
E_{b}\left(\mathcal{Z}^{2 n} x, \mathcal{Z} y\right) & =E_{b}(0,0) \\
& =0 \\
& \leq k_{x} E_{b}\left(\mathcal{Z}^{2 n-1} x, y\right)
\end{aligned}
$$

Case II. If $y=1$, then $\mathcal{Z} y=0$.

$$
E_{b}\left(\mathcal{Z}^{2 n} x, \mathcal{Z} y\right)=E_{b}(0,0)=0 \text { and } E_{b}\left(\mathcal{Z}^{2 n-1} x, y\right)=E_{b}(0,1)=1 .
$$

Hence, $E_{b}\left(\mathcal{Z}^{2 n} x, \mathcal{Z} y\right) \leq k_{x} E_{b}\left(\mathcal{Z}^{2 n-1} x, y\right)$. As a result, all the conditions of the above theorem are satisfied, and ' 0 ' is the unique fixed point where $0 \in \mathbb{S}_{1} \cup \mathbb{S}_{2}$.

Example 3. Let $M=\mathbb{R}$. Define $E_{b}(x, y): M \times M \rightarrow \mathbb{R}_{0}^{+}$and $s: M \times M \rightarrow[1, \infty)$ as $E_{b}(x, y)=(x-y)^{2}$ and $s(x, y)=x+y+1$. Then, $E_{b}$ is a complete $E_{b}$-metric on $M$.

Let $\mathbb{S}_{1}=\left[0, \frac{1}{2}\right], \mathbb{S}_{2}=\left[\frac{1}{3}, 1\right]$. Define $\mathcal{Z}: \mathbb{S}_{1} \cup \mathbb{S}_{2} \rightarrow \mathbb{S}_{1} \cup \mathbb{S}_{2}$ by:

$$
\mathcal{Z} x= \begin{cases}\frac{2}{5}, & \text { if } 0 \leq x \leq \frac{1}{3} \\ \frac{2}{3}(1-x), & \text { if } \frac{1}{3}<x \leq 1\end{cases}
$$

Initially, we have to prove that $\mathcal{Z}$ is a cyclic map.

If $x=0 \in \mathbb{S}_{1}$, then $\mathcal{Z} 0=\frac{2}{5} \in \mathbb{S}_{2}$.

If $x=\frac{1}{2} \in \mathbb{S}_{1}$, then $\mathcal{Z} \frac{1}{2}=\frac{2}{3}\left(1-\frac{1}{2}\right)=\frac{1}{3} \in \mathbb{S}_{2}$.

Similarly, if $x=\frac{1}{3} \in \mathbb{S}_{2}$, then $\mathcal{Z}_{3} \frac{1}{3}=\frac{2}{5} \in \mathbb{S}_{1}$.

If $x=1 \in \mathbb{S}_{2}$, then $\mathcal{Z} 1=\frac{2}{3}(1-1)=0 \in \mathbb{S}_{1}$.

Hence, $\mathcal{Z}\left(\mathbb{S}_{1}\right) \subseteq \mathbb{S}_{2}$ and $\mathcal{Z}\left(\mathbb{S}_{2}\right) \subseteq \mathbb{S}_{1}$. Thus, $\mathcal{Z}$ is a cyclic map.

Fix any $x \in\left[0, \frac{1}{2}\right]$. Let $x=0$, then, we find that:

$$
\mathcal{Z} x=\frac{2}{5}
$$




$$
\begin{aligned}
& \mathcal{Z}^{2} x=\mathcal{Z}(\mathcal{Z} x)=\mathcal{Z}\left(\frac{2}{5}\right)=\frac{2}{5} \\
& \mathcal{Z}^{3} x=\mathcal{Z}\left(\mathcal{Z}^{2} x\right)=\mathcal{Z}\left(\frac{2}{5}\right)=\frac{2}{5}
\end{aligned}
$$

Thus, $\mathcal{Z}^{n} x=\frac{2}{5}$. Therefore, $\mathcal{Z}^{2 n} x=\mathcal{Z}^{2 n-1} x=\frac{2}{5}$.

Case I. If $y=0$.

$$
\begin{gathered}
\mathcal{Z} y=\frac{2}{5}, \\
E_{b}\left(\mathcal{Z}^{2 n} x, \mathcal{Z} y\right)=E_{b}\left(\frac{2}{5}, \frac{2}{5}\right)=0, \\
E_{b}\left(\mathcal{Z}^{2 n-1} x, y\right)=E_{b}\left(\frac{2}{5}, 0\right)=\frac{4}{25} .
\end{gathered}
$$

Therefore, for $k_{x} \in(0,1)$,

$$
E_{b}\left(\mathcal{Z}^{2 n} x, \mathcal{Z} y\right) \leq k_{x} E_{b}\left(\mathcal{Z}^{2 n-1} x, y\right) .
$$

Case II. If $y=\frac{1}{2}$.

$$
\begin{gathered}
\mathcal{Z} y=\frac{2}{3}\left(1-\frac{1}{2}\right)=\frac{1}{3} \\
E_{b}\left(\mathcal{Z}^{2 n} x, \mathcal{Z} y\right)=E_{b}\left(\frac{2}{5}, \frac{1}{3}\right)=\frac{1}{225}=0.004, \\
E_{b}\left(\mathcal{Z}^{2 n-1} x, y\right)=E_{b}\left(\frac{2}{5}, \frac{1}{2}\right)=\frac{1}{100}=0.01 .
\end{gathered}
$$

Therefore, for $k_{x}=\frac{1}{2} \in(0,1)$,

$$
E_{b}\left(\mathcal{Z}^{2 n} x, \mathcal{Z} y\right) \leq k_{x} E_{b}\left(\mathcal{Z}^{2 n-1} x, y\right) .
$$

Case III. If $0<y<\frac{1}{2}$.

Subcase A. If $0<y \leq \frac{1}{3}$.

$$
\begin{gathered}
\mathcal{Z} y=\frac{2}{5}, \\
E_{b}\left(\mathcal{Z}^{2 n} x, \mathcal{Z} y\right)=E_{b}\left(\frac{2}{5}, \frac{2}{5}\right)=0, \\
E_{b}\left(\mathcal{Z}^{2 n-1} x, y\right)=E_{b}\left(\frac{2}{5}, y\right)=\left(\frac{2}{5}-y\right)^{2},
\end{gathered}
$$

Thus, $E_{b}\left(\mathcal{Z}^{2 n} x, \mathcal{Z} y\right) \leq k_{x} E_{b}\left(\mathcal{Z}^{2 n-1} x, y\right)$, for $k_{x} \in(0,1)$.

Subcase B. If $\frac{1}{3} \leq y<\frac{1}{2}$.

$$
\mathcal{Z} y=\frac{2}{3}(1-y)
$$




$$
\begin{aligned}
E_{b}\left(\mathcal{Z}^{2 n} x, \mathcal{Z} y\right) & =E_{b}\left(\frac{2}{5}, \frac{2}{3}(1-y)\right) \\
& =\left(\frac{2}{5}-\frac{2}{3}(1-y)\right)^{2} \\
& =\left(\frac{2}{5}-\frac{2}{3}+\frac{2}{3} y\right)^{2} \\
& =\left(\frac{-4}{15}+\frac{2}{3} y\right)^{2} \\
& =\frac{4}{9}\left(y-\frac{2}{5}\right)^{2} . \\
\text { Thus, } \frac{4}{9}\left(y-\frac{2}{5}\right)^{2} \leq k_{x}\left(y-\frac{2}{5}\right)^{2} \text { for } k_{x}=\frac{1}{2} \in(0,1) . & E_{b}\left(\frac{2}{5}, y\right)=\left(\frac{2}{5}-y\right)^{2} .
\end{aligned}
$$

Hence, in all cases, the extended cyclic orbital condition is satisfied, and $\frac{2}{5}$ is the unique fixed point of $\mathcal{Z}$.

Special cases: If we take,

1. $s(x, y)=k$ for $k>1$, then the above theorem reduces to the $b$-metric space.

2. $s(x, y)=k$ for $k=1$, then the above theorem reduces to the main result of Karpagam et al. [7].

\section{An Extended Cyclic Orbital $\mathcal{F}$-Contraction}

In this section, we join and build a connection between $\mathcal{F}$-contraction and cyclic orbital contraction. Thereby, we build unique fixed point theorems in the setting of $E_{b}$-metric space $\left(M, E_{b}\right)$.

Definition 5. Let $E_{b}$ be a continuous functional in complete $E_{b}$-metric space $\left(M, E_{b}\right)$, and suppose that $\mathbb{S}_{1}$ and $\mathbb{S}_{2}$ are non-empty subsets of an $E_{b}$-metric space $\left(M, E_{b}\right)$. Let $\mathcal{Z}: \mathbb{S}_{1} \cup \mathbb{S}_{2} \rightarrow \mathbb{S}_{1} \cup \mathbb{S}_{2}$ be a cyclic map such that for some $x \in \mathbb{S}_{1}$, there exists $\tau>0$ such that $\forall x, y \in M$ satisfying $E_{b}(\mathcal{Z} x, \mathcal{Z} y)>0$, the following holds:

$$
\tau+F\left(E_{b}\left(\mathcal{Z}^{2 n} x, \mathcal{Z} y\right)\right) \leq F\left(E_{b}\left(\mathcal{Z}^{2 n-1} x, y\right)\right)
$$

where $n \in \mathbb{N}, y \in \mathbb{S}_{1}$ such that for each $x_{0} \in M, \lim _{n, m \rightarrow \infty} s\left(x_{n}, x_{m}\right)<1$; here, $x_{n}=\mathcal{Z}^{n} x_{0}, \quad n=1,2,3 \ldots$ Then, $\mathcal{Z}$ is called an extended cyclic orbital $\mathcal{F}$-contraction.

Theorem 2. Let $E_{b}$ be a continuous functional in complete $E_{b}$-metric space $\left(M, E_{b}\right)$. Let $\mathcal{Z}: \mathbb{S}_{1} \cup \mathbb{S}_{2} \rightarrow \mathbb{S}_{1} \cup \mathbb{S}_{2}$ be an extended cyclic orbital $\mathcal{F}$-contraction. Then, $\mathbb{S}_{1} \cap \mathbb{S}_{2}$ is non-empty and $\mathcal{Z}$ has a unique fixed point in $\mathbb{S}_{1} \cap \mathbb{S}_{2}$

Proof. Suppose there exists an $x \in \mathbb{S}_{1}$ (say $x_{0}$ satisfying (5)).

Since for any $n \in \mathbb{N}$, there is the possibility that either $n$ or $n+1$ is even, we have:

$$
F\left(E_{b}\left(\mathcal{Z}^{n} x_{0}, \mathcal{Z}^{n+1} x_{0}\right)\right) \leq F\left(E_{b}\left(x_{0}, x_{1}\right)\right)-n \tau
$$

Therefore:

$$
\lim _{n \rightarrow \infty} F\left(E_{b}\left(x_{n}, x_{n+1}\right)\right)=-\infty
$$

Since $F \in \mathcal{F}$,

$$
\lim _{n \rightarrow \infty} E_{b}\left(x_{n}, x_{n+1}\right)=0 .
$$


By (6) for all $n \in \mathbb{N}$, we obtain:

$$
\begin{aligned}
E_{b}\left(x_{n}, x_{n+1}\right)^{k} F\left(\left(E_{b}\left(x_{n}, x_{n+1}\right)\right)\right)-F\left(E_{b}\left(x_{0}, x_{1}\right)\right) & \leq-\left(E_{b}\left(x_{n}, x_{n+1}\right)\right)^{k} n \tau . \\
& \leq 0
\end{aligned}
$$

Considering (7) and (8) and letting $n \rightarrow \infty$ in (9), we have:

$$
\left.\lim _{n \rightarrow \infty}\left(n E_{b}\left(x_{n}, x_{n+1}\right)\right)^{k}\right)=0 .
$$

Since (10) holds, there exists $n_{1} \in \mathbb{N}$, such that $n\left(E_{b}\left(x_{n}, x_{n+1}\right)\right)^{k} \leq 1$ for all $n \geq n_{1}$ or:

$$
E_{b}\left(x_{n}, x_{n+1}\right) \leq \frac{1}{n^{\frac{1}{k}}}
$$

for all $n \geq n_{1}$. Using (11) and the triangular inequality, we get from $m>n>n_{1}$,

$$
\begin{aligned}
E_{b}\left(x_{n}, x_{m}\right) \leq & s\left(x_{n}, x_{m}\right)\left[E_{b}\left(x_{n}, x_{n+1}\right)+E_{b}\left(x_{n+1}, x_{m}\right)\right] \\
\leq & s\left(x_{n}, x_{m}\right) E_{b}\left(x_{n}, x_{n+1}\right)+s\left(x_{n}, x_{m}\right) s\left(x_{n+1}, x_{m}\right)\left[E_{b}\left(x_{n+1}, x_{n+2}\right)+E_{b}\left(x_{n+2}, x_{m}\right)\right] \\
\leq & s\left(x_{n}, x_{m}\right) E_{b}\left(x_{n}, x_{n+1}\right)+s\left(x_{n}, x_{m}\right) s\left(x_{n+1}, x_{m}\right) E_{b}\left(x_{n+1}, x_{n+2}\right)+\ldots \\
& +s\left(x_{n}, x_{m}\right) s\left(x_{n+1}, x_{m}\right) s\left(x_{n+2}, x_{m}\right) \ldots s\left(x_{m-2}, x_{m}\right) s\left(x_{m-1}, x_{m}\right) E_{b}\left(x_{m-1}, x_{m}\right) \\
\leq & s\left(x_{1}, x_{m}\right) s\left(x_{2}, x_{m}\right) \ldots . s\left(x_{n}, x_{m}\right) E_{b}\left(x_{n}, x_{n+1}\right) \\
& +s\left(x_{1}, x_{m}\right) s\left(x_{2}, x_{m}\right) \ldots . s\left(x_{n+1}, x_{m}\right) E_{b}\left(x_{n+1}, x_{n+2}\right)+\ldots \\
& +s\left(x_{1}, x_{m}\right) s\left(x_{2}, x_{m}\right) \ldots . s\left(x_{m-1}, x_{m}\right) E_{b}\left(x_{m-1}, x_{m}\right) .
\end{aligned}
$$

Note that this series:

$$
\sum_{n=1}^{\infty} E_{b}\left(x_{n}, x_{n+1}\right) \prod_{i=1}^{n} s\left(x_{i}, x_{m}\right) \text { converges. }
$$

Since,

$$
\begin{aligned}
\sum_{n=1}^{\infty} E_{b}\left(x_{n}, x_{n+1}\right) \prod_{i=1}^{n} s\left(x_{i}, x_{m}\right) & \leq \sum_{n=1}^{\infty} \frac{1}{n^{\frac{1}{k}}} \prod_{i=1}^{n} s\left(x_{i}, x_{m}\right) \\
& \leq \sum_{n=1}^{\infty} \frac{1}{n^{\frac{1}{k}}} \text {, which is convergent. }
\end{aligned}
$$

Let:

$$
\begin{aligned}
& S=\sum_{n=1}^{\infty} E_{b}\left(x_{n}, x_{n+1}\right) \prod_{i=1}^{n} s\left(x_{i}, x_{m}\right) . \\
& S_{n}=\sum_{j=1}^{n} E_{b}\left(x_{j}, x_{j+1}\right) \prod_{i=1}^{j} s\left(x_{i}, x_{m}\right) .
\end{aligned}
$$

Thus, for $m>n$, the above inequality implies:

$$
E_{b}\left(x_{n}, x_{m}\right) \leq S_{m-1}-S_{n-1} .
$$

Letting $n \rightarrow \infty$, we conclude that $\left\{x_{n}\right\}$ is a Cauchy sequence. Hence, $\left\{\mathcal{Z}^{n} x\right\}$ is a Cauchy sequence, and thus, there exists a $\rho \in \mathbb{S}_{1} \cap \mathbb{S}_{2}$ such that $\left\{\mathcal{Z}^{n} x\right\} \rightarrow \rho$. Now, note that $\left\{\mathcal{Z}^{2 n} x\right\}$ is a sequence in $\mathbb{S}_{1}$ and $\left\{\mathcal{Z}^{2 n-1} x\right\}$ is a sequence in $\mathbb{S}_{2}$ and both converge to $\rho$. Since $\mathbb{S}_{1}$ and $\mathbb{S}_{2}$ are closed, $\rho \in \mathbb{S}_{1} \cap \mathbb{S}_{2}$. Hence, $\mathbb{S}_{1} \cap \mathbb{S}_{2}$ is non-empty.

To prove $\rho=\mathcal{Z} \rho$, suppose $\rho \neq \mathcal{Z} \rho$ then from the triangular inequality,

$$
E_{b}(\rho, \mathcal{Z} \rho) \leq s(x, y)\left[E_{b}\left(\rho, \mathcal{Z}^{2 n} x\right)+E_{b}\left(\mathcal{Z}^{2 n} x, \mathcal{Z} \rho\right)\right] .
$$


Letting $n \rightarrow \infty, E_{b}\left(\rho, \mathcal{Z}^{2 n} x\right) \rightarrow 0$.

Since $\mathcal{Z}^{2 n-1} x \rightarrow \rho$ and from the continuity of $\mathcal{Z}, \lim _{n \rightarrow \infty}\left(\mathcal{Z}^{2 n} x, \mathcal{Z} \rho\right)=0$.

From $(14), E_{b}(\rho, \mathcal{Z} \rho)=0$. Therefore, $\rho=\mathcal{Z} \rho$. Hence, $\rho$ is a fixed point of $\mathcal{Z}$.

To prove the uniqueness of $\rho$, suppose there exists an $\varrho \in \mathbb{S}_{1} \cap \mathbb{S}_{2}$, such that $\varrho \neq \rho$ and such that $\mathcal{Z} \varrho=\varrho$.

From (4),

$$
\begin{gathered}
\tau+F\left(E_{b}\left(\mathcal{Z}^{2} \varrho, \mathcal{Z} \rho\right)\right) \leq F\left(E_{b}(\mathcal{Z} \varrho, \rho)\right) \\
\Rightarrow \tau+F\left(E_{b}(\mathcal{Z} \varrho, \rho)\right) \leq F\left(E_{b}(\mathcal{Z} \varrho, \rho)\right) \\
\Rightarrow \tau \leq F\left(E_{b}(\mathcal{Z} \varrho, \rho)\right)-F\left(E_{b}(\mathcal{Z} \varrho, \rho)\right)=0,
\end{gathered}
$$

which is a contradiction. Thus, $\varrho=\rho$. This completes the proof.

Example 4. Let $M=\left\{\frac{1}{2^{n-1}} ; n \in \mathbb{N}\right\} \cup\{0\}$. Define $E_{b}: M \times M \rightarrow \mathbb{R}_{0}^{+}$by $E_{b}(x, y)=(x-y)^{2}$ and $s: M \times M \rightarrow[1, \infty)$ as $s(x, y)=x+y+1$. Then, $E_{b}$ is a complete $E_{b}$-metric on $M$.

Let $\mathbb{S}_{1}=\left\{\frac{1}{2^{2 n-1}} ; n \in \mathbb{N}\right\} \cup\{0\}$ and $\mathbb{S}_{2}=\left\{\frac{1}{2^{2 n}} ; n \in \mathbb{N}\right\} \cup\{0\}$. Define $\mathcal{Z}: \mathbb{S}_{1} \cup \mathbb{S}_{2} \rightarrow \mathbb{S}_{1} \cup \mathbb{S}_{2}$ by:

$$
\mathcal{Z}(x)=\left\{\begin{array}{cl}
\left\{\frac{1}{2^{n}}\right\}, & \text { if } x \in\left\{\frac{1}{2^{n-1}} ; n \in \mathbb{N}\right\} \\
0, & \text { if } x=0 .
\end{array}\right.
$$

Clearly, $\mathcal{Z}\left(\mathbb{S}_{1}\right) \subseteq \mathbb{S}_{2}$ and $\mathcal{Z}\left(\mathbb{S}_{2}\right) \subseteq \mathbb{S}_{1}$. Thus, $\mathcal{Z}$ is a cyclic map.

Fix any $x \in \mathbb{S}_{1}$, and let $x=\frac{1}{2^{2 n-1}} ; \mathcal{Z}^{2 n} x=\frac{1}{2^{4 n-1}} ; \mathcal{Z}^{2 n-1} x=\frac{1}{2^{4 n-2}}$.

Case I. For every $y \in \mathbb{S}_{1} \backslash\{0,1\}$.

$$
\begin{aligned}
\text { Let } y=\frac{1}{2^{2 m-1}} ;(m>n \geq 1) \\
T y=\frac{1}{2^{2 m}} \\
E_{b}\left(\mathcal{Z}^{2 n} x, \mathcal{Z} y\right)=E_{b}\left(\frac{1}{2^{4 n-1}}, \frac{1}{2^{2 m}}\right) \\
=\left(\frac{1}{2^{4 n-1}}-\frac{1}{2^{2 m}}\right)^{2} \\
=\left(\frac{2^{2 m}-2^{4 n-1}}{2^{4 n+2 m-1}}\right)^{2} \\
E_{b}\left(\mathcal{Z}^{2 n-1} x, y\right)=E_{b}\left(\frac{1}{2^{4 n-2}}, \frac{1}{2^{2 m-1}}\right) \\
=\left(\frac{1}{2^{4 n-2}}-\frac{1}{2^{2 m-1}}\right)^{2} \\
=\left(\frac{2^{2 m-1}-2^{4 n-2}}{2^{4 n+2 m-3}}\right)^{2}
\end{aligned}
$$


Define the function $F: \mathbb{R}_{0}^{+} \rightarrow \mathbb{R}$ by $F(x)=\ln x$, for all $x \in \mathbb{R}_{0}^{+}$and $\tau>0$.

$$
\begin{aligned}
F\left(E_{b}\left(\mathcal{Z}^{2 n} x, \mathcal{Z} y\right)\right)-F\left(E_{b}\left(\mathcal{Z}^{2 n-1} x, y\right)\right) & =2\left(\ln \frac{2^{2 m}-2^{4 n-1}}{2^{4 n+2 m-1}}-\ln \frac{2^{2 m-1}-2^{4 n-2}}{2^{4 n+2 m-3}}\right) \\
& =2 \ln \left(\frac{2^{2 m}-2^{4 n-1}}{2^{4 n+2 m-1}} \times \frac{2^{4 n+2 m-3}}{2^{2 m-1}-2^{4 n-2}}\right) \\
& =2 \ln \left(\frac{2^{2 m}-2^{4 n-1}}{2^{2 m-1}-2^{4 n-2}} \times 2^{-2}\right) \\
& =2 \ln \left(\frac{2^{2 m}-2^{4 n-1}}{2^{2 m+1}-2^{4 n}}\right) \\
& =2 \ln \left(\frac{2^{2 m}-2^{4 n-1}}{2\left(2^{2 m}-2^{4 n-1}\right)}\right) \\
& =2 \ln \frac{1}{2} \\
& <-1
\end{aligned}
$$

Case II. If $y=0$,

$$
\begin{aligned}
E_{b}\left(\mathcal{Z}^{2 n} x, \mathcal{Z} y\right) & =E_{b}\left(\frac{1}{2^{4 n-1}}, 0\right) \\
& =\left(\frac{1}{2^{4 n-1}}\right)^{2} \\
E_{b}\left(\mathcal{Z}^{2 n-1} x, y\right) & =E_{b}\left(\frac{1}{2^{4 n-2}}, 0\right) \\
& =\left(\frac{1}{2^{4 n-2}}\right)^{2}
\end{aligned}
$$

Thus, we have,

$$
\begin{aligned}
F\left(E_{b}\left(\mathcal{Z}^{2 n} x, \mathcal{Z} y\right)\right)-F\left(E_{b}\left(\mathcal{Z}^{2 n-1} x, y\right)\right) & =2\left(\ln \frac{1}{2^{4 n-1}}-\ln \frac{1}{2^{4 n-2}}\right) \\
& =2 \ln \left(\frac{1}{2^{4 n-1}} \times \frac{2^{4 n-2}}{1}\right) \\
& =2 \ln \left(2^{-1}\right) \\
& =2\left(\ln \frac{1}{2}\right) \\
& <-1
\end{aligned}
$$

Hence, $\mathcal{Z}$ is an extended cyclic orbital $\mathcal{F}$-contraction for $\tau=1$. Thus, all the conditions of the above theorem are satisfied, and ' 0 ' is the unique fixed point.

Special cases: If we take,

1. $s(x, y)=k$ for $k>1$, then the above theorem reduces to the $b$-metric space.

2. $s(x, y)=k$ for $k=1$, then the above theorem reduces to the metric space.

\section{Applications to the Existence of Solutions for the Fredholm Integral Equation}

Theorem 3. Let $M$ be the set of $\mathbb{C}[a, b]$ of real continuous functions on $[a, b]$, and let $E_{b}: M \times M \rightarrow[0, \infty)$ be given by:

$$
E_{b}(x, y)=\sup _{t \in[a, b]}|x(t)-y(t)|^{2} \text {, with } s(x, y)=|x(t)|+|y(t)|+1,
$$


where s : $M \times M \rightarrow[1, \infty)$.

Easily, we can prove that $M$ is a complete $E_{b}$-metric space. Consider the Fredholm integral equation as,

$$
x(t)=\int_{a}^{b} N(t, p, x(p)) d p+f(t) \text { for all } t, p \in[a, b]
$$

where $f:[a, b] \rightarrow \mathbb{R}$ and $N:[a, b] \times[a, b] \times \mathbb{R} \rightarrow \mathbb{R}$ are continuous functions.

Let $\mathbb{S}_{1}=\mathbb{S}_{2}=M=\mathbb{C}([a, b], \mathbb{R})$. It is clear that $\mathbb{S}_{1}$ and $\mathbb{S}_{2}$ are closed subsets of $\left(M, E_{b}\right)$.

Define $\mathcal{Z}: \mathbb{S}_{1} \cup \mathbb{S}_{2} \rightarrow \mathbb{S}_{1} \cup \mathbb{S}_{2}$ by:

$$
\mathcal{Z} x(t)=\int_{a}^{b} N(t, p, x(p)) d p+f(t) \text { for all } t, p \in[a, b] ;
$$

where the function $f:[a, b] \rightarrow \mathbb{R}$ and $N:[a, b] \times[a, b] \times \mathbb{R} \rightarrow \mathbb{R}$ are continuous.

Clearly, $\mathcal{Z}\left(\mathbb{S}_{1}\right) \subset \mathbb{S}_{2}$ and $\mathcal{Z}\left(\mathbb{S}_{2}\right) \subset \mathbb{S}_{1}$. Thus, $\mathcal{Z}$ is a cyclic map on $\mathbb{S}_{1} \cup \mathbb{S}_{2}$.

Next, assume that the following condition holds well.

$$
|N(t, p, x(p))-N(t, p, \mathcal{Z} x(p))| \leq \frac{1}{2}|x(p)-\mathcal{Z} x(p)| \text { for each } t, p \in[a, b] \text { and } x \in M .
$$

Then, the integral Equation (15) has a solution.

We have to prove that the operator $\mathcal{Z}$ satisfies the conditions of Theorem 2.2.

For $x \in \mathbb{S}_{1}$, consider,

$$
\begin{aligned}
\left|\mathcal{Z}^{2} x(t)-\mathcal{Z} x(t)\right|^{2} & =|\mathcal{Z}(\mathcal{Z}(x(t)))-\mathcal{Z} x(t)|^{2} \\
& \leq\left(\int_{a}^{b}|N(t, p, \mathcal{Z} x(p))-N(t, p, x(p))|\right)^{2} d p \\
& \leq \int_{a}^{b} \frac{1}{2}|\mathcal{Z} x(s)-x(s)|^{2} d p \\
& \leq \frac{1}{4} E_{b}(\mathcal{Z} x, x)
\end{aligned}
$$

which implies:

$$
E_{b}\left(\mathcal{Z}^{2} x, \mathcal{Z} x\right) \leq \frac{1}{4} E_{b}(\mathcal{Z} x, x), \text { since } k_{x}=\frac{1}{4} \in(0,1) .
$$

Thus, all the conditions of Theorem.1 follow by the hypothesis. Therefore, the operator $\mathcal{Z}$ has a fixed point, i.e., the Fredholm integral equation has a solution.

Theorem 4. Let $M$ be the set of all continuous real valued functions defined on $[a, b]$, i.e., $M=\mathbb{C}([a, b], \mathbb{R})$.

Define $E_{b}: M \times M \rightarrow \mathbb{R}$ by $E_{b}(\mathbb{U}, \mathbb{V})=\sup |\mathbb{U}(t)-\mathbb{V}(t)|^{2}, \quad t \in[a, b]$ with $s(\mathbb{U}, \mathbb{V})=|\mathbb{U}(t)|+$ $|\mathbb{V}(t)|+1$, where $s: M \times M \rightarrow[1, \infty)$.

Note that $\left(M, E_{b}\right)$ is a complete $E_{b}$-metric space.

Consider the Fredholm integral equation as,

$$
\mathbb{U}(t)=\int_{a}^{b} \mathcal{M}(t, p, \mathbb{U}(p)) d p+f(t) \forall t, p \in[a, b],
$$

where $f:[a, b] \rightarrow \mathbb{R}$ and $\mathcal{M}:[a, b] \times[a, b] \times \mathbb{R} \rightarrow \mathbb{R}$ are continuous functions.

Let $\mathbb{S}_{1}=\mathbb{S}_{2}=X=\mathbb{C}([a, b], \mathbb{R})$. It is clear that $\mathbb{S}_{1}$ and $\mathbb{S}_{2}$ are closed subsets of $\left(M, E_{b}\right)$.

Define $\mathcal{Z}: \mathbb{S}_{1} \cup \mathbb{S}_{2} \rightarrow \mathbb{S}_{1} \cup \mathbb{S}_{2}$ by $\mathcal{Z}(\mathbb{U}(t))=\int_{a}^{b} \mathcal{M}(t, p, \mathbb{U}(p)) d p+f(t) \forall t, p \in[a, b] ;$ where $f:[a, b] \rightarrow \mathbb{R}$ and $\mathcal{M}:[a, b] \times[a, b] \times \mathbb{R} \rightarrow \mathbb{R}$ are continuous functions. 
Clearly, $\mathcal{Z}\left(\mathbb{S}_{1}\right) \subset \mathbb{S}_{2}$ and $\mathcal{Z}\left(\mathbb{S}_{2}\right) \subset \mathbb{S}_{1}$. Thus, $\mathcal{Z}$ is a cyclic map on $\mathbb{S}_{1} \cup \mathbb{S}_{2}$. Hereafter, assume that the following condition holds.

$$
|\mathcal{M}(t, p, \mathbb{U}(p))-\mathcal{M}(t, p, \mathcal{Z} \mathbb{U}(p))| \leq\left|e^{-\frac{\tau}{2}}\right| \mathbb{U}(p)-\mathcal{Z} \mathbb{U}(p) \mid
$$

for each $t, p \in[a, b], \mathbb{U} \in M$ and $\tau>0$.

Then, the integral Equation (18) has a solution.

We will prove now that the operator $\mathcal{Z}$ satisfies the conditions of Theorem 3.2.

For some $\mathbb{U}(t) \in A$, consider:

$$
\begin{aligned}
\left|\mathcal{Z}^{2} \mathbb{U}(t)-\mathcal{Z} \mathbb{U}(t)\right|^{2} & =|\mathcal{Z}(\mathcal{Z}(\mathbb{U}(t)))-\mathcal{Z} \mathbb{U}(t)|^{2} \\
& \leq\left(\int_{a}^{b} \mathcal{M}(t, p, \mathcal{Z} \mathbb{U}(p))-\int_{a}^{b} \mathcal{M}(t, p, \mathbb{U}(p))\right)^{2} d p \\
& \leq\left(e^{\frac{-\tau}{2}}|\mathcal{Z} \mathbb{U}(p)-\mathbb{U}(p)|\right)^{2} d p \\
& \leq e^{-\tau} E_{b}(\mathcal{Z} \mathbb{U}(t), \mathbb{U}(t))
\end{aligned}
$$

which implies $E_{b}\left(\mathcal{Z}^{2} \mathbb{U}(t), \mathcal{Z} \mathbb{U}(t)\right) \leq e^{-\tau} E_{b}(\mathcal{Z} \mathbb{U}(t), \mathbb{U}(t))$ Thus,

$$
\begin{aligned}
& \ln \left(E_{b}\left(\mathcal{Z}^{2} \mathbb{U}(t), \mathcal{Z} \mathbb{U}(t)\right)\right) \leq \ln e^{-\tau}+\ln \left(E_{b}(\mathcal{Z} \mathbb{U}(t), \mathbb{U}(t))\right) \\
& \Rightarrow \ln \left(E_{b}\left(\mathcal{Z}^{2} \mathbb{U}(t), \mathcal{Z} \mathbb{U}(t)\right)\right) \leq-\tau+\ln \left(E_{b}(\mathcal{Z} \mathbb{U}(t), \mathbb{U}(t))\right), \\
& \Rightarrow \tau+\ln \left(E_{b}\left(\mathcal{Z}^{2} \mathbb{U}(t), \mathcal{Z} \mathbb{U}(t)\right)\right) \leq \ln \left(E_{b}(\mathcal{Z} \mathbb{U}(t), \mathbb{U}(t))\right) .
\end{aligned}
$$

Therefore, all the conditions of Theorem. 2 are satisfied for $F\left(t_{1}\right)=\ln t_{1}, t_{1}>0$. Therefore, the operator $\mathcal{Z}$ has a fixed point. Hence, the Fredholm integral equation has a solution.

\section{Conclusions}

Since Wardowski's characterization of the Banach contraction mapping theorem, many characterizations of contraction type mapping theorems have appeared in the literature. In this article, we introduced the concepts of extended cyclic orbital contraction and extended cyclic orbital- $\mathcal{F}$-contraction in the setting of $E_{b}$-metric space. Thereafter, we proposed a simple and efficient solution for a Fredholm integral equation by using the technique of the fixed point in the setting of the $E_{b}$-metric space. We hope that the results contained in this article will build new connections for those who are working in $\mathcal{F}$-contraction (or its generalizations), cyclic orbital contraction (or its generalizations) and its applications to differential, integral and functional equations.

Author Contributions: All authors contributed equally and significantly in writing this article. All authors read and approved the final manuscript.

Funding: The corresponding author acknowledges with thanks Deanship of Scientific Research, Taibah University, Al Madina Al Munawara, KSA for financial support.

Conflicts of Interest: The authors declare no conflict of interest.

\section{References}

1. Fredholm, E.I. Sur une classe d'equations fonctionnelles. Acta Math. 1903, 27, 365-390. [CrossRef]

2. Rus, M.D. A note on the existaence of positive solution of Fredholm integral equations. Fixed Point Theory 2004, 5, 369-377.

3. Berenguer, M.I.; Munoz, M.V.F.; Guillem, A.I.G.; Galan, M.R. Numerical Treatment of Fixed Point Applied to the Nonlinear Fredholm Integral Equation. Fixed Point Theory Appl. 2009, 2009, 1-8. [CrossRef]

4. Pathak, H.K.; Khan, M.S.; Tiwari, R. A common fixed point theorem and its application to nonlinear integral equations. Comput. Math. Appl. Arch. 2007, 53, 961-971. [CrossRef] 
5. Rasham, T.; Shoaib, A.; Hussain, N.; Arshad, M.; Khan, S.U. Common fixed point results for new Ciric-type rational multivalued F-contraction with an application. J. Fixed Point Theory Appl. 2018, 20, 45. [CrossRef]

6. Kirk, W.A.; Srinavasan, P.S.; Veeramani, P. Fixed Points for mapping satisfying cyclical contractive conditions. Fixed Point Theory 2003, 4, 79-89.

7. Karpagam, S.; Agrawal, S. Best proximity point theorems for cyclic orbital Meir-Keeler contraction maps. Nonlinear Anal. Theory Methods Appl. 2011, 74, 1040-1046. [CrossRef]

8. Wardowski, D. Fixed point theory of a new type of contractive mappings in complete metric spaces. Fixed Point Theory Appl. 2012, 2012, 94. [CrossRef]

9. Kumari, P.S.; Panthi, D. Cyclic compatible contraction and related fixed point theorems. Fixed Point Theory Appl. 2016, 2016, 28. [CrossRef]

10. Menger, K. Untersuchungen uber allgeneine Metrik? Math. Ann. 1928, 100, 75-163. [CrossRef]

11. Kumari, P.S.; Sarma, I.R.; Rao, J.M. Metrization theorem for a weaker class of uniformities. Afrika Matematika 2016, 27, 667-672. [CrossRef]

12. Kumari, P.S.; Panthi, D. Cyclic contractions and fixed point theorems on various generating spaces. Fixed Point Theory Appl. 2015, 2015, 153. [CrossRef]

13. Sarma, I.R.; Rao, J.M.; Kumari, P.S.; Panthi, D. Convergence Axioms on Dislocated Symmetric Spaces. Abstr. Appl. Anal. 2014, 2014, 1-7. [CrossRef]

14. Kamran, T.; Samreen, M.; Ain, Q. A generalization of $b$-metric space and some fixed point theorems. Mathematics 2017, 5, 19. [CrossRef]

15. Alqahtani, B.; Karapinar, E.; Ozturk, A. On $(\alpha, \psi)$-K-contractions in the extended $b$-metric space. Filomat 2018, 32. Available online: http://journal.pmf.ni.ac.rs/filomat/index.php/filomat/article/view/8154 (accessed on 13 October 2018).

16. Alqahtani, B.; Fulga, A.; Karapinar, E. Non-Unique Fixed Point Results in Extended B-Metric Space. Mathematics 2018, 6, 68. [CrossRef]

17. Alqahtani, B.; Fulga, A.; Karapinar, E. Common fixed point results on extended b-metric space. J. Inequal. Appl. 2018, 2018, 158. [CrossRef] [PubMed]

18. Rhoades, B.E. Comments on some Fixed Point Theorems. Results Fixed Point Theory Appl. 2018, 2018 , 1-10. [CrossRef]

19. Dlala, E.; Belahcen, A.; Arkkio, A. A Fast Fixed-Point Method for Solving Magnetic Field Problems in Media of Hysteresis. IEEE Trans. Magn. 2008, 44, 1214-1217. [CrossRef]

(C) 2018 by the authors. Licensee MDPI, Basel, Switzerland. This article is an open access article distributed under the terms and conditions of the Creative Commons Attribution (CC BY) license (http:// creativecommons.org/licenses/by/4.0/). 